Ann. Biol. anim. Bioch. Biophys., I973, 13 (I), I47-I50.

NOTE

\title{
INFLUENCE DE L'ADMINISTRATION DE MÉTABISULFITE DE POTASSIUM SUR LA RESERVE HÉPATIQUE DE VITAMINE A, CHEZ LE RAT SOUMIS A UN RÉGIME GARENCÉ EN CETTE VITAMINE
}

\author{
Denise HUGOT et J. LECLERC \\ Station de Recherches sur la Qualité des Aliments de l'Homme, \\ Centre de Rechérches de Dijon, I. N. R. A., \\ 21034 Dijon Cedex
}

\section{RÉSUMÉ}

Chez le Rat soumis à un régime alimentaire carencé en vitamine $\mathrm{A}$, l'introduction de $2 \mathrm{p}$. Ioo de métabisulfite de potassium đans le régime ralentit l'épuisement de la réserve hépatique de cette vitamine.

Au cours d'une étude sur la toxicité chronique des sulfites, Fitzhugh et al. (1946) ont signalé, parmi les symptômes pathologiques observés chez des rats soumis à des régimes contenant 0,5 à 2 p. 100 de métabisulfite de sodium, une dépigmentation des incisives. Cette lésion pouvait faire penser à une carence en vitamine A. Irving et al. (1952) ont effectué par la suite une étude histologique des incisives prélevées chez un certain nombre de rats utilisés par Firzhugh. Ils ont noté une atrophie des odontoblastes, une invasion de la pulpe par l'épithélium odontogénique et une métaplasie de l'épithélium du canal naso-lacrymal, - symptômes évidents, d'après les auteurs, d'une carence en vitamine $\mathrm{A}$.

D'autre part, chez des rats qui recevaient comme boisson une solution de métabisulfite de potassium à $\mathrm{I}, 2 \mathrm{~g}$ par litre, soit pendant 20 mois avec un régime alimentaire équilibre, soit pendant 4 mois avec un régime ne contenant pas de vitamine A, CAUSERET el al. (r965) n'ont observé aucune différence significative entre les teneurs en vitamine $\mathrm{A}$ du foie de ces animaux et celles de témoins qui recevaient de l'eau distillée. Dans ces conditions expérimentales, l'ingestion de sulfite ne semble pas provoquer ou aggraver une carence en vitamine A. Mais les doses administrées étaient nettement inférieures à celles à partir desquelles IRving et al. ont obtenu des symptômes suggérant une carence induite en vitamine A. Nous avons donc repris l'étude de l'évolution de la teneur hépatique en vitamine A, au cours de la carence, chez des rats recevant 
une dose élevée de sulfite. Dans ces conditions, les animaux refusent généralement l'eau fortement sulfitée, alors qu'ils acceptent la même dose de sulfite introduite dans le régime : en conséquence, ce dernier mode d'administration a été choisi.

L'étude a porté sur I33 rats mâles Wistar, provenant de l'élevage du laboratoire et mis en expérience au sevrage, à l'âge de 3 semaines. Vingt et un d'entre eux ont été sacrifiés immédiatement, afin de déterminer leur réserve hépatique en vitamine $A$. Les autres ont été répartis en 2 lots qui ont reçu un régime carencé, en vitamine $A$, identique par ailleurs à celui utilisé dans les expériences antérieures de notre groupe. La composition centésimale est la suivante :

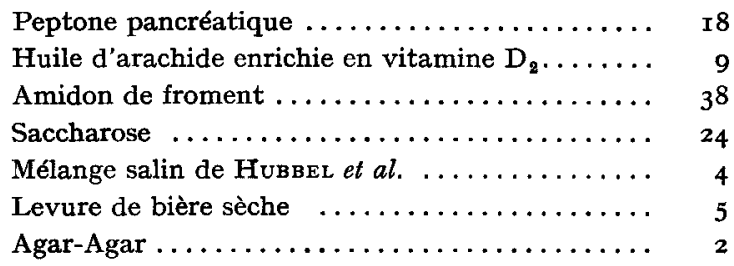

Ce régime a été distribué sous forme de bouillie obtenue par addition de $350 \mathrm{ml}$ d'eau distillée à $\mathbf{~} \mathrm{kg}$ du mélange précédent.

Dans le cas du lot 2 , du métabisulfite de potassium, à la dose de 2 p. roo du régime sec, a été ajouté juste avant la distribution aux animaux, afin de limiter la destruction de la thiamine dans le régime (Morgan et al., 1935). En outre, pour tenir compte des possibilités de destruction de cette même vitamine par $\mathrm{SO}_{2}$ dans le tube digestif (LHuissier, 1966), l'eau de boisson du lot 2 a été enrichie en vitamine $B_{1}$ à raison de $2 \mathrm{mg}$ par litre.

Dans le lot I " témoin " et dans le lot 2 "sulfite ", des animaux ont été sacrifiés après ro, 20, 30 et 40 jours d'expérience. La vitamine A a été extraite du foie par l'éther de pétrole, puis dosée par spectrophotométrie à $328 \mathrm{~m} \mu$.

Les résultats sont indiqués dans le tableau 1.

Chez tous les animaux, la réserve hépatique de vitamine A diminue progressivement, mais plus ou moins vite selon les lots :

- au to $^{\mathrm{e}}$ jour, elle est la même dans les 2 lots bien que le poids du foie soit plus grand chez les sujets qui reçoivent du métabisulfite de potassium (lot 2),

- à partir du $20^{\circ}$ jour, elle est plus élevée dans le lot 2 que dans le lot I (témoin), cette augmentation étant due pour une part à un accroissement du poids du foie, et pour une autre, à une concentration vitaminique plus forte.

Notre travail ne fournit aucune indication sur le mécanisme du phénomène, ni même sur son interprétation. On peut en particulier se demander si le sulfite se comporte comme un "facteur d'économie "de la vitamine A, ou s'il perturbe le métabolisme de cette vitamine au niveau du foie.

Par ailleurs, comme nous l'avons rappelé, les sulfites peuvent détruire la thiamine in vitro dans les aliments et même in vivo dans le tube digestif en entraînant ainsi une diminution de l'apport de cette vitamine à l'organisme. Or Johnson et Baumann (1948) ont montré que la réserve hépatique de vitamine $A$ diminue plus lentement chez des rats carencés lorsque le régime est très pauvre en thiamine. Le régime administré à nos animaux n'assurait qu'un rapport peu élevé de cette vitamine (environ Io $\mu \mathrm{g}$ par jour). Toutefois, les sujets du lot 2 recevaient un important supplément de thiamine dans l'eau de boisson (environ $30 \mu \mathrm{g}$ par jour) : il est donc peu probable que la quantité de vitamine $B_{1}$ fournie à leur organisme ait été moins élevée que chez les animaux du lot $\mathrm{I}$ et qu'un phénomène analogue à celui signalé par JoHNSon et BaumanN soit à l'origine de nos résultats.

Ces résultats, pas plus que ceux de nos recherches antérieures, ne confirment l'existence d'une carence en vitamine A provoquée par l'administration de sulfite. 
RÉSERVE HÉPATIQUE DE VITAMINES A DU RAT

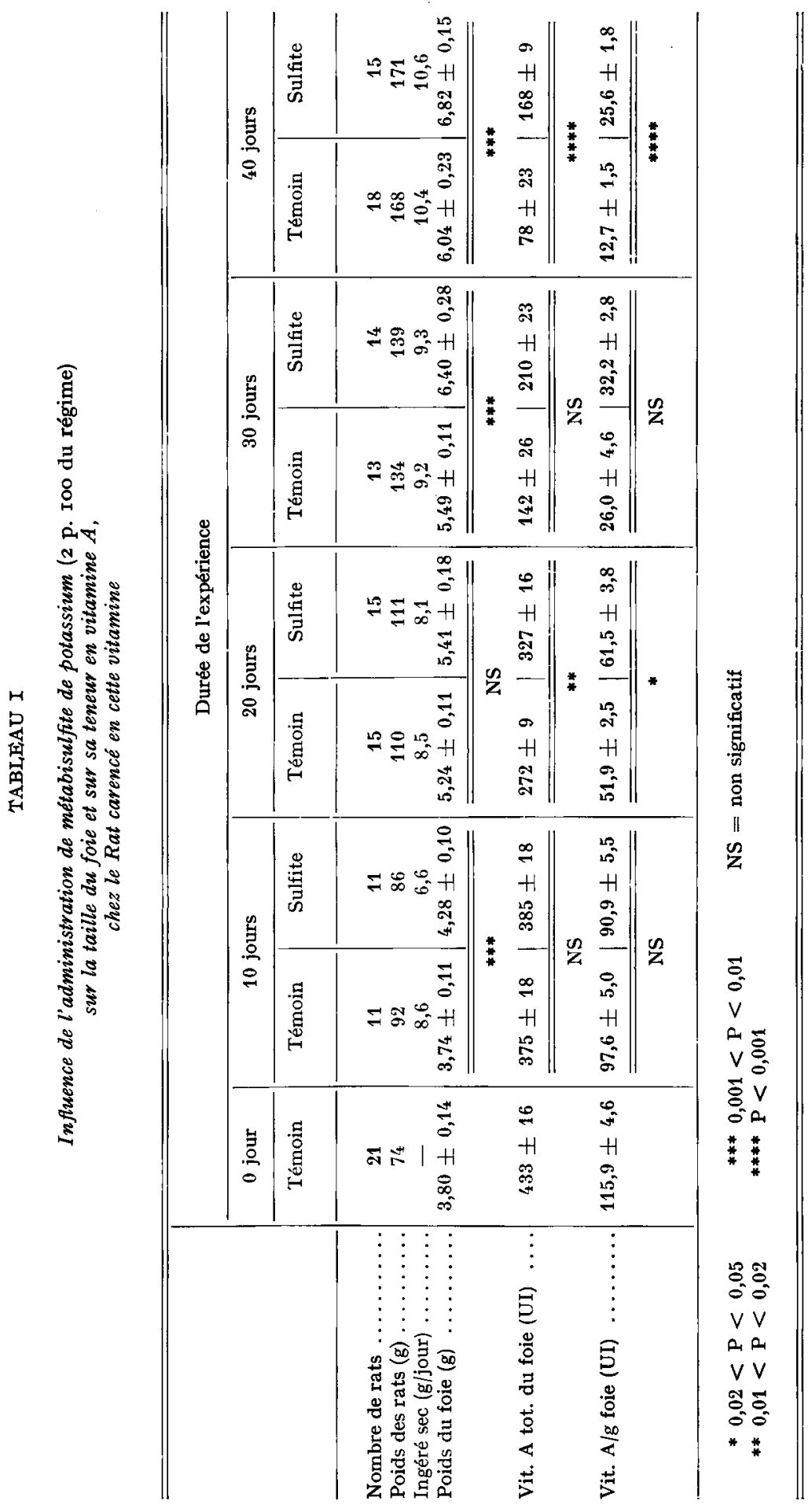




\section{SUMMARY}

\section{EFFECT OF POTASSIUM METABISULFITE ADMINISTRATION ON VITAMIN A HEPATIC STORAGE IN THE RAT EATING A DIET DEFICIENT IN THIS VITAMIN}

In the rat eating a vitamin-A deficient diet, introducing $2 \mathrm{p}$. Ioo potassium metabisulfite in the diet, slows down drainage of the hepatic store of this vitamin.

\section{RÉFÉRENCE,S BIBLIOGRAPHIQUES}

Causeret J., Hugot D., Lhuissier M., Biette E., Leclerc J., I965. L'utilisation des sulfites en technologie alimentaire : quelques aspects toxicologiques et nutritionnels. Fruits, 20, Io9-II5.

Fitzhugh O. G., Knudesen L. F., Nelson A. A., 1946. The chronic toxicity of sulfites. J. Pharmacol., 86, 37-48.

Irving J. T., Pindborg J. J., Fitzhugh O. G., Weitnmann J. P., Schour I., I952. Symptoms of vitamin $A$ and $E$ deficiencies in the incisor of the rat fed sodium sulfite. J. Dent. Res., 31, 8I5-824.

Johnson R. M., BaUmann C. A., I948. Relative significance of growth and metabolic rate upon the utilization of vitamin A by the Rat. J. Nutr., 35, 703-715.

Lhuissier M., I966. Influence des sulfites sur l'efficacité biologique de la vitamine $B_{1}$. C. R. Acad. nat. Méd., 150, 242-245.

Morgan A. F., Kimmel L., Field A., Nichols P. F., I935. The vitamin content of sultania (Thompson seedless) grapes and raisins. J. Nutr., 9, 369-38z. 